\title{
Cranberry juice consumption may reduce biofilms on uroepithelial cells: pilot study in spinal cord injured patients
}

\author{
G Reid*,1,2,3 J Hsiehl $^{4}$, P Potter ${ }^{4}$, J Mighton ${ }^{2}$, D Lam ${ }^{1}$, D Warren ${ }^{4}$ and J Stephenson ${ }^{2}$ \\ ${ }^{1}$ Lawson Health Research Institute, London, Ontario, Canada; ${ }^{2}$ Department of Microbiology and Immunology, The \\ University of Western Ontario, Ontario, Canada; ${ }^{3}$ Department of Surgery, Division of Urology, The University of \\ Western Ontario, Ontario, Canada; ${ }^{4}$ Parkwood Hospital Site, St. Joseph's Health Care London, London, Ontario, \\ Canada
}

\begin{abstract}
Study design: A pilot study of 15 spinal cord injured patients.
Objective: To determine whether alteration of fluid intake and use of cranberry juice altered the bacterial biofilm load in the bladder.

Setting: London, Ontario, Canada.

Methods: Urine samples were collected on day 0 (start of study), on day 7 following each patient taking one glass of water three times daily in addition to normal diet, and on day 15 following each patient taking one glass of cranberry juice thrice daily. One urine sample was sent for culture and a second processed to harvest, examine by light microscopy and Gram stain non-squamous uroepithelial cells to generate bacterial adhesion per 50 cells data.

Results: The results showed that cranberry juice intake significantly reduced the biofilm load compared to baseline $(P=0.013)$. This was due to a reduction in adhesion of Gram negative $(P=0.054)$ and Gram positive $(P=0.022)$ bacteria to cells. Water intake did not significantly reduce the bacterial adhesion or biofilm presence.

Conclusion: The findings provide evidence in support of further, larger clinical trials into the use of functional foods, particularly cranberry juice, to reduce the risk of UTI in a patient population highly susceptible to morbidity and mortality associated with drug resistant uropathogens.
\end{abstract}

Sponsorship: This study was funded by Ocean Spray Cranberries, Lakeville, MA, USA. Spinal Cord (2001) 39, 26-30

Keywords: cranberry juice; bacteria; biofilms; bladder infection

\section{Introduction}

The annual incidence of spinal cord injury (SCI), not including those who die at the scene of an accident, is approximately 40 per million population. At any given time, there are over 200000 patients with spinal cord injury in North America, and all of them will suffer from symptomatic UTI at some time following disability. ${ }^{1}$ Indeed, in some patients, this infection can be fatal. Antibiotics are effective at clearing symptoms of UTI, but recurrences are high and bladder colonization is inevitable, primarily because of failure of the controlled micturition defence mechanism and need for intermittent or permanent catheterization. Problems arise when the infecting organisms are able to resist the antibiotics, or when they develop into dense biofilm structures which are not eradicated easily by traditional drug therapy. ${ }^{2,3}$

*Correspondence: G Reid, Lawson Health Research Institute, 268 Grosvenor Street, London, Ontario, N6A 4V2, Canada
Cranberry juice supplements have been shown to improve the health of the urinary tract by inhibiting binding of uropathogenic bacteria to the bladder mucosa, lowering the urinary $\mathrm{pH}$ and acting as a flushing agent. The theory that cranberry juice reduces the risk of infection is based upon in vitro and clinical evidence. ${ }^{4-6}$ However, recent publications have questioned whether or not cranberry juice really does prevent UTI or influence bladder health. ${ }^{7,8}$

A study undertaken by our group ${ }^{9}$ has shown that 3 days intake of cranberry supplement leads to production of urine which is more acidic and less able to support the binding of uropathogens to surfaces. The effect of these supplements on the maintenance of an infection free bladder in hospitalized patients with spinal cord or neurogenic bladder disease caused by other ailments has not previously been tested. The hypothesis is that intake of cranberry will discourage growth and colonization of the bladder by uropathogenic bacteria, thereby maintaining an infection free state without the need for antibiotic therapy. 


\section{Materials and methods}

\section{Patients}

Fifteen patients with spinal cord injury (quadriplegia and paraplegia) were recruited for the study. Each was over 18 years of age (mean age $=42.3 \pm 14.9$ years), and signed an informed consent approved by the Ethics Review Board of The University of Western Ontario. The patients had a previous history of UTI, were not presently receiving antibiotics, had normal renal function (serum creatinine $>110 \mathrm{umol} / 1$, upper limit 90 umol/1), did not have pyelonephritis, were not pregnant, were not receiving prednisone or immunosuppressive drugs, had no history of autonomic dysreflexia as a result of UTI in past 12 months, had no known urinary abnormality such as cancer or stone disease, and did not have symptomatic UTI at time of study. Patients were asked to refrain from intake of additional cranberry supplements or additional vitamin $\mathrm{C}$ in the 3 days prior to and for the duration of the study.

\section{Protocol}

The patients consented to take a $250 \mathrm{ml}$ glass of water in addition to their normal diet, at breakfast, lunchtime and dinner time for 7 days. On the ninth day, for a further 7 days, each patient took a 250-ml glass of cranberry juice (Ocean Spray Cranberries, Lakeville, MA, USA) at the three meal times. The nurse monitored compliance and enquired about any perceived or adverse events on days 7 and 15. Each patient provided a urine sample on day 0,7 and 15 using the same collection protocol. This specimen was sent for culture by standard microbiology laboratory, and used to harvest uroepithelial cells. The cells were washed in phosphate buffered saline (PBS, pH 7.1), air dried onto slides, Gram stained and analyzed under oil immersion. Bacteria attached to 50 cells viewed at random were enumerated, and Gram positive and Gram negative bacterial counts were differentiated. In addition, cells with biofilms present $(>10$ bacteria closely associated on the surface of a cell) were noted and recorded as a percentage of the total cells.

\section{Results}

The results are presented in Tables 1 and 2, and Figure 1. Statistically, the cranberry juice intake caused a significant reduction in biofilm load compared to baseline $(P=0.013)$ and to water treatment $(P=0.028)$. The reductions were due to lower Gram negative bacterial adhesion counts on the uroepithelial cells which fell from a mean of 9.9 at day 7 to 1.7 after cranberry intake for 1 week $(P=0.033)$, and to lower Gram positive counts from 2.4 to $0.7(P=0.173)$. The Gram positive bacterial counts fell significantly from day 0 to day $15(P=0.022)$, as did the Gram negative counts $(P=0.054)$. Increased water intake did not reduce the number of cells covered with biofilms $(P=0.36)$.

Patient 6 developed a symptomatic UTI with $E$. coli on day 7 following water intake, but this resolved with cranberry juice and no antibiotics. In half of the urine culture results (Table 2) there is a correlation between the findings for adhesion and biofilm counts. In patient 2, the highest biofilms were Gram positive cocci, yet the urine culture showed P. mirabilis; in patient 3 the culture showed beta hemolytic streptococci but higher adhesion by Gram negative rods; in patient 4 biofilms were found without urine growth; in

Table 1 This presents the results obtained for biofilms (percentage of cells with a biofilm present) on uroepithelial cells. The cells were gram stained and the mean number of Gram positive $(G+)$ and Gram negative $(G-)$ organisms found per cell is reported with standard deviation $(\mathrm{SD})$

\begin{tabular}{|c|c|c|c|c|c|c|c|c|c|}
\hline \multirow[b]{2}{*}{ Patient } & \multicolumn{3}{|c|}{ Day 0} & \multicolumn{3}{|c|}{ Day 7} & \multicolumn{3}{|c|}{ Day 15} \\
\hline & $G+$ & $G-$ & Biofilm & $G+$ & $G-$ & Biofilm & $G+$ & $G-$ & Biofilm \\
\hline 2 & 2.52 & 1.12 & 14 & 0.04 & 0.02 & 0 & 0.14 & 1.16 & 6 \\
\hline 3 & 0.34 & 0.7 & 4 & 0.16 & 2.12 & 4 & 0.04 & 0.08 & 0 \\
\hline 4 & 0 & 1.34 & 6 & 4.32 & 2.94 & 24 & 0 & 0.32 & 2 \\
\hline 5 & 7.42 & 21.22 & 72 & 8.5 & 17.4 & 68 & 2.96 & 4.84 & 20 \\
\hline 6 & 1.28 & 1.5 & 8 & 0.24 & 4.62 & 16 & 0.2 & 2.34 & 4 \\
\hline 7 & 0.58 & 0.26 & 0 & 19 & 2.96 & 0.1 & & & \\
\hline 8 & 0.36 & 0.08 & 2 & 0.72 & 0.64 & 2 & 0 & 0.1 & 0 \\
\hline 9 & & & & 0 & 32.5 & 22.7 & 0 & 0 & 0 \\
\hline 10 & 0.6 & 1.6 & 8 & 0 & 11.6 & 18 & 0 & 4.2 & 12 \\
\hline 11 & 0 & 0.33 & 0 & 0 & 5.63 & 0 & 0 & 1.45 & 0 \\
\hline 12 & 11.2 & 0 & & 3.6 & 0 & & 1.8 & 0 & \\
\hline 16 & 0 & 7.1 & 24 & 0 & 0.31 & 0 & 0 & 0.48 & 0 \\
\hline 17 & 17.1 & 14.2 & 26.7 & 0 & 0 & 0 & 4.2 & 0 & 0.8 \\
\hline 18 & 1.77 & 6.12 & 23 & 0 & 5.77 & 15 & 0 & 2.96 & 15.3 \\
\hline 21 & 0 & 52.4 & 75.7 & 0 & 62.4 & 91.1 & 0 & 5.6 & 13 \\
\hline Mean & 3.08 & 7.71 & 20.26 & 2.44 & 9.93 & 10.06 & 0.67 & 1.68 & 5.62 \\
\hline SD & 5.2 & 14.3 & 25.5 & 5.2 & 16.9 & 28.9 & 1.34 & 1.97 & 7.03 \\
\hline
\end{tabular}


Table 2 Results for urine cultures ${ }^{\mathrm{a}}$ from male (M) and female (F) spinal cord patients

\begin{tabular}{|c|c|c|c|c|c|}
\hline Patient & Age & Sex & Day 0 & Day 7 & Day 15 \\
\hline 2 & 50 & M & 80000 P. mirabilis & No growth & 60000 mixed culture \\
\hline 3 & 32 & $\mathrm{~F}$ & 25000 streptococci & 50000 streptococci & NSG \\
\hline 4 & 26 & M & No growth & No growth & No growth \\
\hline 5 & 48 & M & 100000 streptococci & 100000 streptococci & 100000 streptococci \\
\hline 6 & 37 & M & 100000 mixed culture & 100000 E. coli & 100000 streptococci \\
\hline 7 & 31 & M & $80000 P$. aeruginosa & $100000 P$. aeruginosa & \\
\hline 8 & 44 & M & No growth & No growth & No growth \\
\hline 9 & 78 & M & 30000 P. mirabilis & 100000 P. mirabilis & $100000 \mathrm{P}$. mirabilis \\
\hline 10 & 36 & $\mathrm{~F}$ & No growth & NSG & NSG \\
\hline 11 & nd & nd & $\mathrm{NSG}$ & NSG & NSG \\
\hline 12 & 61 & M & 15000 Enterococcus & NSG & NSG \\
\hline 16 & 48 & M & 30000 mixed culture & NSG & NSG \\
\hline 17 & 28 & $\mathrm{~F}$ & 30000 mixed culture & No growth & 80000 mixed culture \\
\hline 18 & 21 & M & $100000 \mathrm{~K}$. pneumoniae & $100000 \mathrm{~K}$. pneumoniae & $100000 \mathrm{~K}$. pneumoniae \\
\hline 21 & 53 & $\mathrm{~F}$ & 100000 E. coli & 100000 E. coli & 60000 E. coli \\
\hline
\end{tabular}

NSG: no significant growth; mixed: more than one pathogen; ${ }^{a}$ colony counts per ml urine; nd: not determined

\section{Effects of Cranberry Juice Consumption on the Adherence of Bacteria to Uroepithelial Cells}

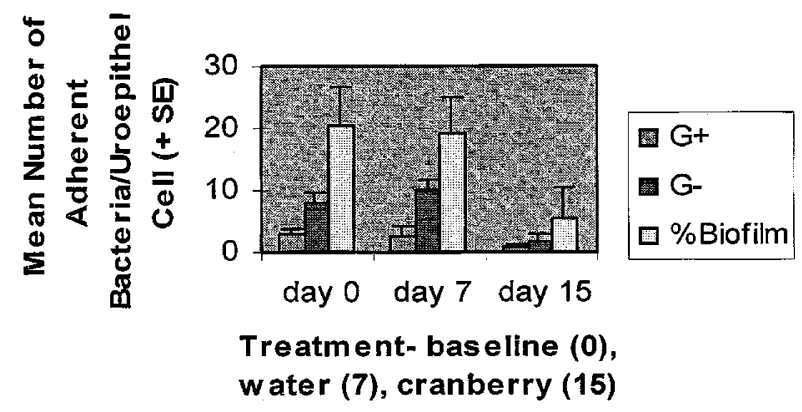

Figure 1 Mean number of adherent Gram positive $(G+)$ and Gram negative $(G-)$ bacteria per uroepithelial cell and percentage of cells covered in biofilms following treatment with water (day 7) then cranberry juice (day 15). Bacterial adhesion compared to baseline day 0

patient 5, high Gram negative biofilm counts were found yet urine culture identified streptococci; in patient 7, biofilms were present on only a few cells yet urine counts were high; in patient 9, Gram negative biofilms corresponded to urine findings; in patient 12 the enterococci culture findings corresponded to adhesion data; in patient 17, the mixed cultures corresponded to adhesion of both Gram positive cocci and Gram negative rods; in patients 18 and 21, the high adhesion counts and culture were both due to Gram negative organisms.

Using urine culture alone as the end point, water treatment reduced pathogen count per ml urine in four cases and increased it in four; cranberry juice treatment reduced pathogen counts in two and increased it in two.

\section{Discussion}

The study, albeit a pilot one, provided a number of interesting findings. There was a clear trend towards reduced bacterial colonization of bladder epithelial cells from patients who drank cranberry juice thrice daily for one week. Apart from patients 2, 6, 8 and 11, juice intake resulted in lower numbers of Gram negative adherent bacteria on day 15 compared to day 0 . Juice intake resulted in lower Gram positive cocci adhesion. The reduction in adhesion with cranberry intake was all the more definitive given that adhesion counts rose with water intake in four cases for Gram positive organisms and seven for Gram negatives, meaning that for cranberry juice to have an effect, the adhesion reduction had to be even greater than it would have been at day 0 .

There is likely some day to day variance in the biofilm counts regardless of treatment effects and we cannot rule this out in the findings. However, once a biofilm has formed, it tends to remain intact, ${ }^{2}$ and so we believe that the differences seen here are more likely due to treatment than day-to-day fluctuations, particularly given that each patient acted as his/her own control and all data was pooled to reduce any variation in one person. Also, the results here showed statistical difference between treatment weeks, while a previous study comparing biofilm counts for eight untreated patients at weekly intervals showed no statistical difference. ${ }^{10}$ We cannot explain why some patients had biofilms on up to $97 \%$ of their cells, while others had them present on $2 \%$. This may be due to the fact that cell populations in the urine in the latter patient contained cells from areas not yet colonized. The fact that some cells had biofilms in a given sample certainly confirms that colonization had taken place somewhere in the bladder.

There was no statistical evidence that increased water intake reduced the bacterial load in the bladder 
or the risk of UTI $(P=0.359)$. Indeed, the one symptomatic $E$. coli case of UTI which occurred, was with patient 5 following the week's water intake protocol. This latter finding supports a previous study which showed, contrary to traditional medical practice of increasing fluid intake to eradicate or prevent UTI, the increase of water produces urine which more readily supports the adhesion of pathogens to surfaces. ${ }^{9}$

The reduction in Gram positive bacterial adhesion was somewhat surprising given that there is no previous data to indicate that cranberry juice inhibits adhesion of these organisms in vitro. Bacterial adhesion to bladder cells can be achieved by electrostatic, hydrophobic and adhesin-receptor ligand mechanisms. ${ }^{3}$ The high counts of adherence in patients 5, 17 and 21 suggest the latter mechanism might be involved. The concept of bacteria attaching to bladder cells and causing UTI stems from in vitro work done 25 years ago. ${ }^{11}$ Since then, it has been assumed that higher adhesion correlates with infection, but in clinical practice this is not necessarily the case as mean counts of $5-10$ bacteria per cell can be found in the bladder cells of infected patients. ${ }^{12}$ Clearly, the levels of adhesion here are very low $(<10$ per cell $)$ in most cases $(85 \%)$ at time zero, and biofilms were present in less than $50 \%$ of cells in $92 \%$ samples, yet significant bacteriuria $(\geqslant 10000$ bacterial per $\mathrm{ml})$ was still detected in the majority $(73 \%)$ of patients at day 0 .

Vaccines are being developed with a view to inhibiting adhesion of highly adherent bacteria. ${ }^{13,14}$ but these are targeting type 1 fimbriated E. coli, and only two out of 15 patients here were infected with this organism, thereby illustrating one disadvantage with the vaccine approach. Also, it is doubtful whether a vaccine can detach already adherent bacteria, while perhaps functional food components in urine can alter the surface interface to allow this to happen. In most of the patients, lower levels of adhesion occurred, making it more feasible that inhibition and detachment of bacteria could be achieved with functional foods, such as cranberry juice. The use of a probiotic preparation containing Lactobacillus rhamnosus GR-1 and L. fermentum RC-14, has been shown in one case study to eradicate enterococci from the bladder and vagina. ${ }^{15}$ Other options such as colonizing the bladder of spinal cord injured patients with avirulent E. coli, such as strain 83972 , appear to hold promise. ${ }^{16}$

The urine culture findings were interesting for a number of reasons (Table 2). Firstly, the laboratory, like many which service hospitals, did not provide accurate bacterial counts per $\mathrm{ml}$, nor did it provide levels below 10000 per ml, or a breakdown of mixed bacterial species. This makes it difficult for physicians to interpret findings. Thus, without symptoms and signs of infection, episodes of bacteriuria are not generally treated with antibiotics in spinal cord injured patients. ${ }^{1}$ The problem of UTI being caused by virulent, invariably multi-drug resistant pathogens other than $E$. coli, namely $P$. aeruginosa, $K$ pneumonia, $P$. mirabilis and enteroccoci is illustrated in this 15 patient population, and appears typical of such hospitalized cases. ${ }^{2,17}$ The culture findings did not reflect the organisms colonizing the patients in half the cases, in agreement with previous studies. ${ }^{2}$ The cranberry treatment did not reduce the urine counts for streptococci (patient 5), K. pneumoniae (patient 14) or P. mirabilis (patient 9) and while the E. coli count was reduced in patient 21 , the clinical significance of the findings remain to be determined. Indeed, the urine culture data showed that neither water or cranberry reduced the number of pathogens present on any consistent basis. Given that urine has been shown to be a poor indicator of colonization and infection in spinal cord injured patients, ${ }^{2,10}$ this finding emphasizes that further studies are required to prove the efficacy of cranberry juice, but also that parameters in addition to urine culture need to be tested.

While this is a pilot study with patient-to-patient differences which make general conclusions inappropriate, there is good evidence to undertake a larger clinical study of cranberry juice to reduce the risk of symptomatic UTI. Given the virulent, drug resistant organisms which cause UTI in these patients, and the high morbidity and mortality rates, alternatives to antibiotics are badly needed.

\section{Acknowledgements}

The cooperation of patients at Parkwood Hospital in London, Ontario is appreciated. The authors have no conflict of interest and hold no shares in Ocean Spray Cranberries.

\section{References}

1 Reid G, Nicolle L. Asymptomatic bacteriuria in spinal cord injured patients and elderly. Urol Clin North Am 1999; 26: $789-$ 795.

2 Reid G et al. Bacterial biofilm formation in the urinary bladder of spinal cord injured patients. Paraplegia 1992; 30: 711-717.

3 Habash M, Reid G. Microbial biofilms: their development and significance for medical device related infections. $J$ Clin Pharmacol 1999; 39: 1-12.

4 Sobota AE. Inhibition of bacterial adherence by cranberry juice: potential use for the treatment of urinary tract infection. $J$ Urol 1984; 131: $1013-1016$.

5 Schmidt DR, Sobota AE. An examination of the anti-adherence activity of cranberry juice on urinary and non-urinary bacterial isolates. Microbios 1988; 55: $171-181$.

6 Avorn $\mathrm{J}$ et al. Reduction of bacteriuria and pyuria after ingestion of cranberry juice. JAMA 1994; 271: $751-754$.

7 Foda MMR et al. Efficacy of cranberry in prevention of urinary tract infection in a susceptible pediatric population. Can J Urol 1995; 2: $98-102$

8 Harkins KJ. What's the use of cranberry juice? Age Ageing 2000; 29: $9-12$

9 Habash M, van der Mei HC, Reid G, Busscher HJ. The effect of water, ascorbic acid and cranberry derived supplementation on human urine and uropathogen adhesion to silicone rubber. Can $J$ Microbiol 1999; 45: 691-694. 
10 Reid $\mathrm{G}$ et al. Bacterial biofilm formation on the bladder epithelium of spinal cord injured patients. II. Toxic outcome on cell viability. Paraplegia 1993; 31: 494-499.

11 Svanborg Eden $\mathrm{C}$ et al. Variable adherence to normal urinarytract epithelial cells of Escherichia coli. Strains associated with various forms of urinary-tract infections. Lancet 1976; 2: $490-$ 492.

12 Reid G, Brooks HJL. In vitro attachment of Escherichia coli to human epithelial cells. NZ Med J 1984; 97: 439-442.

13 Langermann $\mathrm{S}$ et al. Prevention of mucosal Escherichia coli infection by FimH-adhesin-based systemic vaccination. Science 1997; 276: $607-611$.
14 Palaszynski S et al. Systemic immunization with conserved pilusassociated adhesins protects against mucosal infections. Dev Biol Standard 1998; 92: $117-122$.

15 Reid G. Probiotic therapy and functional foods for prevention of UTIS: State of the art and science. Curr Infect Dis Reports 2000; 2: $518-522$.

16 Hull $\mathrm{R}$ et al. Urinary tract infection prophylaxis using Escherichia coli 83972 in spinal cord injured patients. J. Urol. 2000; 163: $872-877$.

17 Reid G, Howard L. Effect on uropathogens of prophylaxis for urinary tract infection in spinal cord injured patients. Spinal Cord 1997; 35: 605-607. 